\title{
PCSK9 immunization using nanoliposomes: preventive efficacy against hypercholesterolemia and atherosclerosis
}

Amir Abbas Momtazi-Borojeni ${ }^{1,2}$, Mahmoud Reza Jaafari3 ${ }^{3,4}$, Mohammad Afshar ${ }^{5,6}$, Maciej Banach ${ }^{7,8}$, Amirhossein Sahebkar ${ }^{4,9,10}$

\author{
${ }^{1}$ Nanotechnology Research Center, Bu-Ali Research Institute, Mashhad University \\ of Medical Sciences, Mashhad, Iran \\ ${ }^{2}$ Department of Medical Biotechnology, Faculty of Medicine, Mashhad University \\ of Medical Sciences, Mashhad, Iran \\ ${ }^{3}$ Nanotechnology Research Center, Pharmaceutical Technology Institute, \\ Mashhad University of Medical Sciences, Mashhad, Iran \\ ${ }^{4}$ Biotechnology Research Center, Pharmaceutical Technology Institute, \\ Mashhad University of Medical Sciences, Mashhad, Iran \\ ${ }^{5}$ Department of Anatomy, Faculty of Medicine, Birjand University of Medical Sciences, \\ Birjand, Iran \\ ${ }^{6}$ Medical Toxicology Research Center, Mashhad University of Medical Sciences, \\ Mashhad, Iran \\ ${ }^{7}$ Department of Hypertension, WAM University Hospital in Lodz, Medical University \\ of Lodz, Lodz, Poland \\ ${ }^{8}$ Polish Mother's Memorial Hospital Research Institute (PMMHRI), Lodz, Poland \\ ${ }^{9}$ Applied Biomedical Research Center, Mashhad University of Medical Sciences, \\ Mashhad, Iran \\ ${ }^{10}$ School of Pharmacy, Mashhad University of Medical Sciences, Mashhad, Iran
}

Submitted: 17 February 2021; Accepted: 2 March 2021

Online publication: 18 March 2021

Arch Med Sci 2021; 17 (5): 1365-1377

DOI: https://doi.org/10.5114/aoms/133885

Copyright @ 2021 Termedia @ Banach

\section{Abstract}

Introduction: The aim of the study was to study a nanoliposomal anti-PCSK9 vaccine as a novel approach for cholesterol lowering via PCSK9 inhibition. Material and methods: An immunogenic peptide construct termed immunogenic fused PCSK9-tetanus (IFPT) was displayed on the surface of liposome nanoparticles (L-IFPT) and mixed into alum adjuvant (L-IFPTA+). The manufactured vaccine formulations IFPT, L-IFPT, L-IFPTA+, IFPTA+, and free nanoliposomes were subcutaneously injected four times with bi-weekly intervals in C57BL/6 mice on a severe atherogenic protocol.

Results: Among the formulations, L-IFPTA+ vaccine was found to elicit the highest IgG response against PCSK9 peptide. The induced PCSK9 antibodies inhibited PCSK9-LDLR interaction through binding to PCSK9 in vaccinated mice. Liver low-density lipoprotein receptor (LDLR) protein was increased in vaccinated mice. L-IFPTA+, L-IFPT and IFPTA+ vaccines reduced total cholesterol by up to $-38.13 \pm 3.8 \%(p=0.006),-23 \pm 4.1 \%(p=0.027)$ and -19.12 $\pm 3 \%(p=0.038)$, and low-density lipoprotein cholesterol (LDL-C) by up to $-57 \pm 7.7 \%(p=0.0003),-41.67 \pm 4.2 \%(p=0.03)$ and $-36.11 \pm 5 \%(p=$ 0.02 ) in hypercholesterolemic mice, respectively, versus control mice after 8 weeks. Long-term assessment indicated that the vaccine formulations could stimulate a long-lasting humoral immune response against PCSK9 peptide, which was associated with a marked reduction of total cholesterol in L-IFPTA+, L-IFPT and IFPTA+ vaccine groups by up to $-82.5 \pm 7.3 \%(p=$ $0.002),-70.54 \pm 6.2 \%(p=0.013)$ and $-72.02 \pm 8.7 \%(p=0.004)$, respectively, and LDL-C by up to $-88.14 \pm 5.6 \%(p=0.002),-55.92 \pm 8.3 \%(p=0.003)$ and $54.81 \pm 9.3 \%(p=0.003)$, respectively, versus the pre-vaccination time point

\author{
Corresponding authors: \\ Amirhossein Sahebkar \\ Biotechnology \\ Research Center \\ Pharmaceutical \\ Technology Institute \\ Mashhad University \\ of Medical Sciences \\ Mashhad 9177948564 \\ Iran \\ E-mail: sahebkara@mums.ac.ir; \\ amir_saheb2000@yahoo.com \\ Mahmoud Reza Jaafari \\ Department of \\ Pharmaceutical \\ Nanotechnology \\ School of Pharmacy \\ Mashhad University \\ of Medical Sciences \\ Mashhad 91775-1365 \\ Iran \\ E-mail: jafarimr@mums.ac.ir
}


adjusted to the control group. Anti-inflammatory Th2 cells and IL-4 cytokine were considerably increased in splenocytes of vaccinated mice.

Conclusions: L-IFPTA+ vaccine can induce long-lasting, functional and safe PCSK9-specific antibodies in hypercholesterolemic C57BL/6 mice, providing a long-term protective impact on dyslipidemia and atherosclerosis.

Key words: PCSK9, immunization, atherosclerosis, dyslipidemia.

\section{Introduction}

Low-density lipoprotein (LDL) cholesterol (LDL-C) lowering is known to be a safe and effective therapeutic approach for atherosclerotic cardiovascular disease [1]. Patients achieving extremely low LDL levels have lower incidence of cardiovascular events as evidenced by several outcome trials [2-5]. The clinical evidence demonstrates that extremely low plasma levels of LDL-C directed by elevated LDLR activity are not correlated with any considerable adverse effects [1].

LDL-C is mainly cleared from the bloodstream by the hepatic LDLR under the control of proprotein convertase subtilisin/kexin 9 (PCSK9). This enzyme plays a crucial role in the metabolism of plasma LDL-C, and PCSK9 inhibitors potently decrease elevated LDL-C concentrations in patients with familial hypercholesterolemia (FH) and/or cardiovascular disease (CVD) who did not achieve optimal LDL-C levels in spite of being on standardof-care treatments, such as high-intensity statin therapy [6-8]. PCSK9 inhibitors are also known to reduce the coronary atherosclerosis burden. Imaging studies performed in the GLAGOV trial showed that adding evolocumab on top of statins augmented the impact of statins in reducing atheroma volume [9].

In recent years, several PCSK9-inhibiting methods have been developed, including gene silencing using RNA interference strategies, and inhibition of PCSK9 binding to LDLR using monoclonal antibodies (mAbs) or small peptides. The use of $m A b s$ is currently the only approved approach for the inhibition of PCSK9-LDLR interaction [10-13]. However, due to their relatively limited in vivo half-lives, long-term administration of mAbs has drawbacks in terms of cost and compliance. Such limitations could be circumvented by active vaccination. More recently, some preclinical studies have shown the effectiveness of peptide-based active vaccination against PCSK9 in mouse models of hypercholesterolemia [14-17]. The main limitation of peptide antigens is their low immunogenicity. Adjuvant systems are commonly used as an effective strategy in vaccine development for improving the immunogenicity of antigenic peptides. Some adjuvants, such as virus-like particles [17], DNA [16], and keyhole limpet hemocyanin $(K L H)[14,15]$, have been used to intensify the immunogenicity of peptide-based antiPCSK9 vaccines; however, their safety and efficiency in human are still unclear.

Tetanus toxin is an approved adjuvant carrier used in human vaccines [18], and we utilized it as a peptide adjuvant covalently linked to PCSK9 antigen to enhance the vaccine immunogenicity. On the other hand, displaying the self-antigens in a repetitive and highly dense structure is an effective strategy for provoking potent antibody responses against self-antigens [19, 20]. Liposome are biocompatible and biodegradable bilayer vesicles that as adjuvant delivery systems have widely been employed in vaccine compositions. These spherical particles are feasible carriers for displaying antigens in peptide-based vaccines [21]. We previously demonstrated that anionic nanoliposomes as a carrier adjuvant could strongly induce a humoral immune response against displayed PCSK9 antigens. It was found that nanoliposomes displaying PCSK9-mimicking peptide linked to tetanus peptide can elicit long-term, strong, safe and specific antibodies against PCSK9 in BALB/C mice [22]. Our further study on C57BL/ 6 mice with severe atherosclerosis indicated that this vaccine setting, in a therapeutic approach, could generate functional and specific antibodies against PCSK9, which was associated with a long-lasting therapeutic effect on hypercholesterolemia and atherosclerosis [23]. Since the atherosclerotic plaque builds up gradually in the artery wall in which fat accumulation (fatty streaks) begins in early childhood and clinically relevant lesions become evident in middle-aged adults [24] and eventually, several years later, clinical manifestations become evident [25], preventing atheroma formation at its very initial stages can be an effective strategy to improve outcomes in individuals at risk of developing atherosclerosis [26]. To this end, the present study aimed to test the preventive efficacy of nanoliposome-displayed PCSK9 as an alternative peptide vaccine for PCSK9 inhibition against dyslipidemia and atherosclerosis in a C57BL/6 mouse model of severe atherosclerosis.

\section{Material and methods}

\section{Preparation of nanoliposomal anti-PCSK9 vaccine}

The lipid-film hydration approach was employed to manufacture liposome nanoparticles formulated 
Table I. Sequence of the immunogenic peptides used in the present study

\begin{tabular}{|lcc|}
\hline Peptide name & Sequence & Immunogenicity \\
\hline PCSK9 & S-I-P-W-N-L-E-R-I-T-P-V-R & B cell epitope \\
\hline Tetanus & A-Q-Y-I-K-A-N-S-K-F-I-G-I-T-E-L & T cell epitope \\
\hline IFPT & ${ }^{*}$ CGGGSIPWNLERITPVRKKAQYIKANSKFIGITEL & \\
\hline
\end{tabular}

*The bold amino acid codes exhibit linker sequences. IFPT - immunogenic fused PCSK9-tetanus.

with 1,2-dimyristoyl-sn-glycero-3-phosphorylglycerol (DMPG), 1,2-dimyristoyl-sn-glycero-3-phosphorylcholine (DMPC), and cholesterol (Chol). To construct vaccine formulation, an immunogenic peptide construct termed immunogenic fused PCSK9-tetanus (IFPT) (Table I) was linked to the nanoliposome surface using DSPE-PEG-maleimide (1,2-distearoyl-sn-glycero-3-phosphoethanolamine- $N$-[maleimide(PEG)-2000]) lipid, and then adsorbed onto Alum adjuvant [further details are described in the previous publication (22)].

\section{Vaccination and atherosclerosis development}

Thirty-five male C57BL/6 mice (6-8 weeks old, weighing $20 \pm 3 \mathrm{~g}$ ) were obtained from the Laboratory Animal Research Center of the Pasteur Institute of Tehran, Iran. All animal treatments were performed in the basis of the Animal Welfare guidelines recommended by the European Parliament [27] and Institutional Ethics Committee and Research Advisory Committee of the Mashhad University of Medical Sciences, Mashhad, Iran. The animals were housed in an air-conditioned room at a constant temperature of $22 \pm 2^{\circ} \mathrm{C}$ with 12 : $12 \mathrm{~h}$ light/dark cycle and fed a standard mouse diet and water ad libitum. At the end of the study, all animals were sacrificed by sodium thiopental (30 mg/kg), intravenously [28, 29].

The mice were randomly sorted into five groups: 1) IFPT vaccine group, 2) IFPT plus Alhydrogel (IFPTA ${ }^{+}$) vaccine group, 3) nanoliposomal IFPT (L-IFPT) vaccine group, 4) L-IFPTA ${ }^{+}$vaccine group, 5) free-nanoliposome (negative control) group. All groups were subcutaneously vaccinated four times with the prepared vaccine formulations $(200 \mu \mathrm{l}$ in both the right and left flanks), once every 2 weeks. To develop atherosclerosis, the vaccinated and control mice were fed an atherogenic chow diet (containing 20\% fat, $1.5 \%$ cholesterol, and $0.5 \%$ cholic acid) and concurrently treated every third day with intraperitoneal administration of poloxamer $407(0.5 \mathrm{~g} / \mathrm{kg})$ for 16 weeks. This model has been shown to produce human-like pathological features of atherosclerosis in mice [30]. To measure titers of anti-PCSK9 antibody and plasma levels of lipid parameters, venous blood was taken at bi-weekly intervals throughout vaccination and post-vaccination periods for 16 weeks.

\section{Analysis of vaccination effects}

To measure the titers of anti-PCSK9 antibodies, plasma samples were isolated and assessed by ELISA technique. Concentrations of plasma PCSK9 in hypercholesterolemic vaccinated $\mathrm{C} 57 \mathrm{BL} / 6$ mice were determined by a PCSK9 ELISA kit (CircuLex, Cy-8078, MBL, Woburn, MA) according to the manufacturer's manual. To verify targeting of mouse PCSK9 by vaccine-generated antibodies, PCSK9 binding to plasma antibodies was evaluated using the modified protocol of the PCSK9 ELISA kit [22]. CircuLex PCSK9-LDLR in vitro binding assay kit (CircuLex, Cy-8150, MBL, Woburn, MA) was utilized to determine the ability of plasma antibodies to suppress the PCSK9-LDLR interaction. Total cholesterol (TC), direct LDL-C, TG (triglyceride), and direct HDL-C (high-density lipoprotein cholesterol) were assessed using commercial kits. To measure expression of liver LDLR protein in vaccinated mice, Western blot analysis and immunohistochemical staining were carried out. The flow cytometry analysis was employed to determine the population of proinflammatory Th1 and anti-inflammatory Th2 cells, $\mathrm{CD}^{+}$cells and their intracellular IFN- $\gamma$ and IL-4 cytokines. At the end of the study, size and severity of atherosclerotic lesions were assessed in the aortic arch of vaccinated mice fed on an atherogenic diet, using hematoxylin and eosin (H\&E) staining. The severity grade of lesions was classified between 1-4 [31]. Further details are described in the previous publication [23].

\section{Statistical analysis}

Unpaired 2-tailed Student's t-test and one-way ANOVA followed by Tukey's post-hoc test were carried out to define the significance of the difference among groups (GraphPad Prism Software, version 7, San Diego, CA). Data were reported as mean \pm SD and lower-upper $95 \%$ confidence interval $(\mathrm{Cl})$ of mean, or median (interquartile range) for normally and non-normally distributed data, respectively. Data with $p<0.05$ were regarded as statistically significant.

\section{Results}

\section{Characterization of nanoliposomes}

Characteristics of the free and IFPT-linked nanoliposome formulations are listed in Table II. The size 
Table II. Physical properties of nanoliposomal formulations

\begin{tabular}{|lccc|}
\hline Formulation & $\begin{array}{c}\text { Z-average }[\mathrm{nm}] \\
\text { mean } \pm \text { SD }(n=3)\end{array}$ & $\begin{array}{c}\text { Zeta potential [mV] } \\
\text { mean } \pm \text { SD }(n=3)\end{array}$ & $\begin{array}{c}\text { PDI } \\
\text { mean } \pm \text { SD }(n=3)\end{array}$ \\
\hline Empty nanoliposome & $125 \pm 4.5$ & $-43.9 \pm 3.8$ & $0.01 \pm 0.001$ \\
\hline IFPT-linked nanoliposome & $155 \pm 5.5$ & $-35.7 \pm 4.2$ & $0.02 \pm 0.008$ \\
\hline PDI - polydispersity index. & & &
\end{tabular}

range of liposome nanoparticles was $120 \mathrm{~nm}$ to $155 \mathrm{~nm}$ in diameter, and the polydispersity index was $<0.1$, indicating nanoparticles with high homogeneity. A negative zeta potential was also identified on the surface of the prepared nanoliposomes.

\section{Nanoliposomal vaccine provokes antibody against peptide PCSK9 in C57BL/6 mice}

Among vaccine formulations, nanoliposomal vaccine displaying IFPT peptide formulated with Alhydrogel (L-IFPTA ${ }^{+}$vaccine) could induce the greatest IgG titers against PCSK9 peptide in the vaccinated mice. Two weeks after the last vaccination (week 8), hypercholesterolemic mice immunized with L-IFPTA ${ }^{+}$formulation indicated 1.41

A

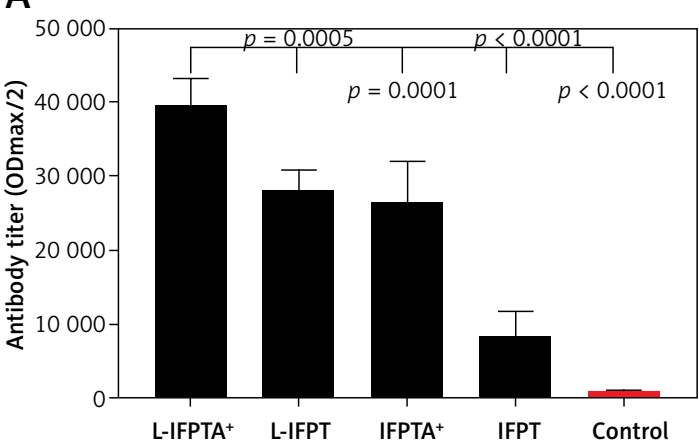

C

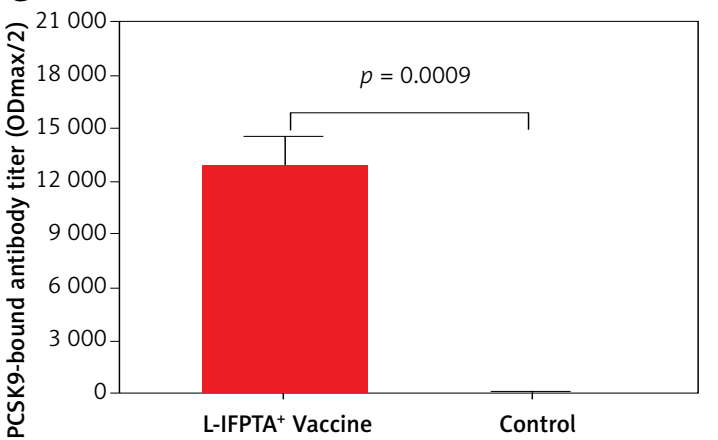

( $p=0.0005), 1.5(p=0.0001)$, and $4.7(p<0.0001)$ fold higher serum antibody titers than mice immunized with the L-IFPT, IFPTA ${ }^{+}$, IFPT formulations, respectively (Figure $1 \mathrm{~A}$ ). Notably, mice immunized by vaccines formulated with liposome nanoparticles showed higher titers of PCSK9 antibody than vaccine formulations without nanoliposome including IFPTA ${ }^{+}$and IFPT. In addition, as demonstrated by a long-term study, PCSK9 antibody in the L-IFPTA ${ }^{+}$vaccine group achieved peak titers at week 8 and stayed constant until week 12 , then exhibited a decreasing trend (Figure 2 A). In conclusion, the findings reveal effective adjuvant potential of liposome nano-carriers to provoke an antibody response against PCSK9 peptide antigen.

B

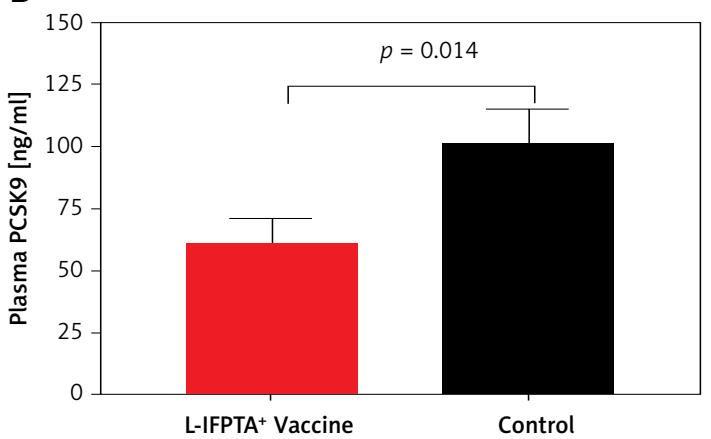

D

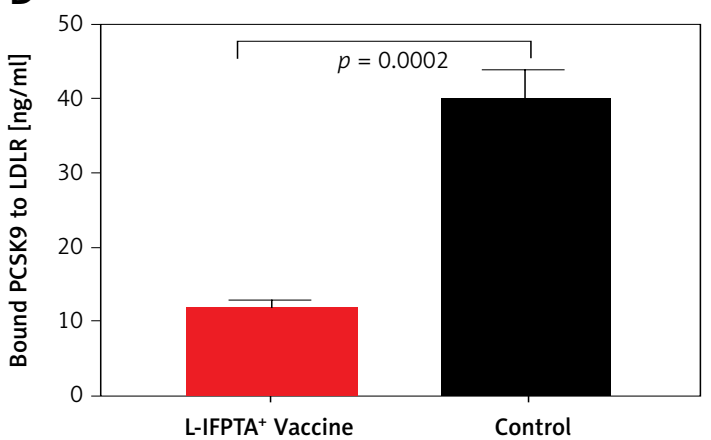

Figure 1. Efficacy of antiPCSK9 vaccines in C57BL/6 mice, 2 weeks after the last vaccination. A - Antibody titers (ODmax/2) against PCSK9 peptide in the plasma of mice vaccinated with various vaccine formulations or mice inoculated with free nanoliposomes as a control. B - Plasma levels of PCSK9 in vaccinated and control groups were $61.37 \pm 5.53 \mathrm{ng} / \mathrm{ml}$ and $101.5 \pm 8.04 \mathrm{ng} / \mathrm{ml}$, respectively. C - Direct detection of antibodies bound to plasma PCSK9 (target engagement) in blood samples from vaccinated and control mice. Increased ODmax/2 is indicative for vaccine-generated PCSK9 antibodies directly binding to PCSK9. D - In vitro PCSK9/LDLR binding assay. Vaccine-generated antiPCSK9 antibodies inhibit PCSK9-LDLR interaction. Plasma sample of L-IFPTA ${ }^{+}$vaccine group decreased PCSK9 binding to LDLR by $69.86 \%$, compared with plasma sample of the control group. Values are means \pm SD, $n=3$ replicates of the pooled samples of 7 mice per group. Significance compared to control values was analyzed by unpaired 2-tailed Student's $t$-test. Statistical differences at $p$-values less than 0.05 were considered to be significant

IFPT - immunogenic fused PCSK9-tetanus, L-IFPT - nanoliposomal IFPT, IFPTA+ - IFPT plus Alhydrogel, L- IFPTA+ - nanoliposomal IFPT plus Alhydrogel. 


\section{Impact of vaccine-provoked PCSK9} antibodies on plasma PCSK9 levels

Vaccine-provoked PCSK9 antibodies in hypercholesterolemic C57BL/6 mice were detected to reduce plasma levels of PCSK9 through specific targeting. Blood concentrations of PCSK9 in the L-IFPTA vaccine and control group were found to be 61.37 $\pm 5.53 \mathrm{ng} / \mathrm{ml}$ and $101.5 \pm 8.04 \mathrm{ng} / \mathrm{ml}$, respectively (Figure $1 \mathrm{~B}$ ). The level of plasma PCSK9 was significantly lower by $40 \%(-40.13 \pm 9.758, p=0.014)$ in vaccinated mice than control mice. To verify specific targeting, an ELISA-based approach was employed [22] to detect plasma PCSK9-bound antibodies. As revealed in Figure $1 \mathrm{C}$, the L-IFPTA ${ }^{+}$vaccine group exhibited high titers of PCSK9-bound antibody, verifying direct binding of vaccine-induced antibodies to plasma PCSK9. Overall, L-IFPTA ${ }^{+}$vaccine can provoke specific antibodies that directly target and eliminate circulating PCSK9 from the bloodstream.

A

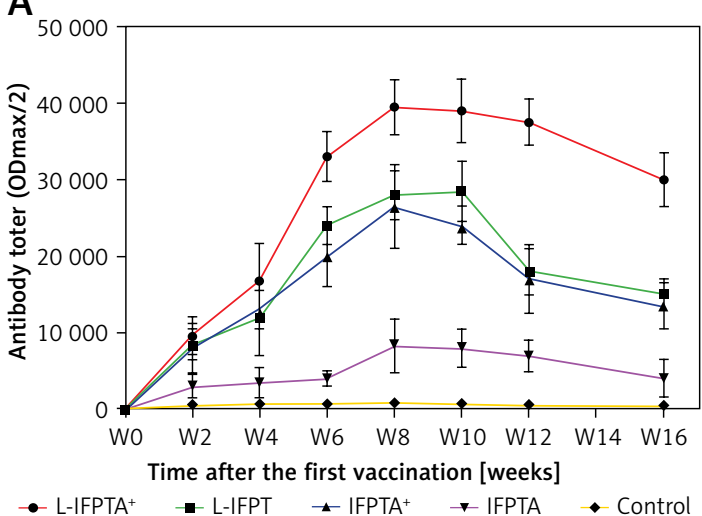

C

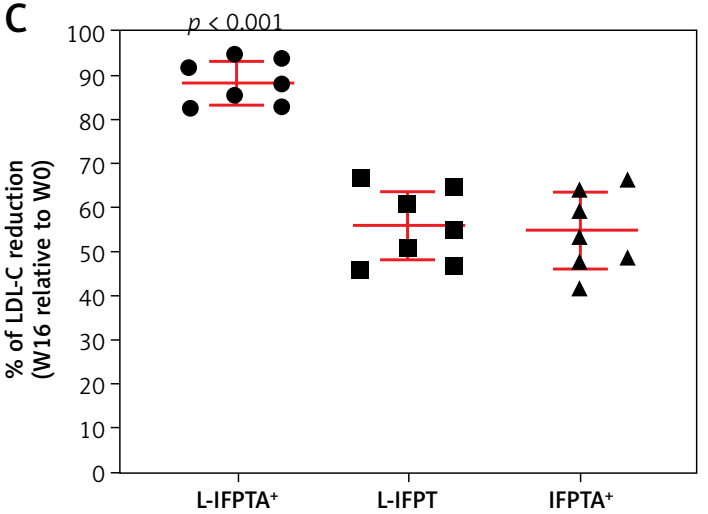

\section{Impact of vaccine-provoked PCSK9} antibodies on PCSK9 function

To determine whether the PCSK9 antibodies can suppress PCSK9 function, the in vitro binding of PCSK9 and LDLR in the presence of plasma PCSK9 antibodies was assayed. It was found that a plasma sample of the L-IFPTA $^{+}$vaccine group could significantly reduce in vitro PCSK9-LDLR binding by $69.86 \%$, in comparison with a plasma sample of the control group (Figure $1 \mathrm{D})$. Overall, the L-IFPTA ${ }^{+}$vaccine induced generation of PCSK9 antibodies in hypercholesterolemic C57BL/6 mice, which can suppress PCSK9-LDLR interaction and thereby inhibit PCSK9 function.

\section{Liposomal vaccines alleviate hypercholesterolemia in C57BL/6 mice}

The liposomal vaccine showed a protective effect against hypercholesterolemia in C57BL/6
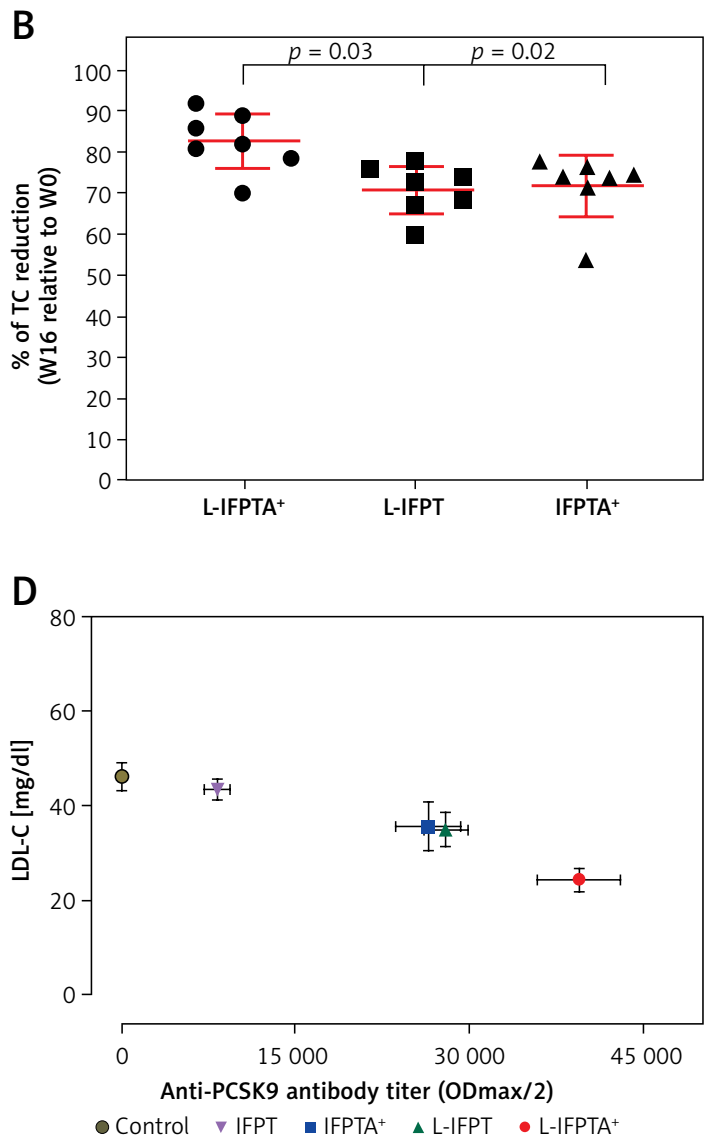

Figure 2. Long-term efficacy of liposomal vaccines in hypercholesterolemic mice. A - Long-term evaluation of antibody titers over 16 weeks after prime vaccination against PCSK9 peptide (ODmax/2). Data are shown as means $\pm \mathrm{SD}$ ( $n=7$ per group). Long-term changes of plasma TC (B) and LDL-C (C) in the vaccinated hypercholesterolemic C57BL/6 mice, at week 16 compared to week 0 (pre-vaccination) adjusted to control group. TC (B) and LDL-C (C) levels were reduced in the L-IFPTA ${ }^{+}$, L-IFPT, and IFPTA+ vaccine group 8 weeks after the last vaccination. Data are shown as means with $95 \%$ confidence intervals ( $n=7$ per group). $\mathbf{D}-$ Correlation plot of circulating LDL-C levels with PCSK9 antibody titers evaluated 2 weeks after the last vaccination (week 8) with L-IFPTA ${ }^{+}$, L-IFPT, and IFPTA vaccine and control liposome. A significant negative correlation was identified among LDL-C and the PCSK9 antibodies in the vaccinated and in control mice $(r=-0.94, p=0.008)$. One-way ANOVA followed by Tukey's post-hoc test was used to determine the significance of the difference among groups. Statistical differences at $p$-values less than 0.05 were considered to be significant 
mice. The L-IFPTA+ vaccine resulted in an increasing trend of plasma TC and LDL-C in hypercholesterolemic C57BL/6 mice, at week 8 (Figure 3). TC was lower in hypercholesterolemic C57BL/6 mice vaccinated with L-IFPTA ${ }^{+}(-38.13 \pm 3.8 \%, 95 \% \mathrm{Cl}$ : $34-41 \%, p=0.006)$, L-IFPT $(-23 \pm 4.1 \%, 95 \% \mathrm{Cl}$ : $19-27 \%, p=0.027)$, and IFPTA ${ }^{+}(-19.12 \pm 3 \%$, $95 \% \mathrm{Cl}: 16-22 \%, p=0.038)$ than in the control group (Figures $3 \mathrm{~A}, \mathrm{E}$ ). LDL-C was reduced in hypercholesterolemic mice vaccinated with L-IFPTA ${ }^{+}$ $(-57 \pm 7.7 \%, 95 \% \mathrm{Cl}: 50-64 \%, p=0.0003)$, L-IFPT $(-41.67 \pm 4.2 \%, 95 \% \mathrm{Cl}: 37-45 \%, p=0.03)$, and IFPTA $^{+}(-36.11 \pm 5 \%$, 95\% Cl: $31-40 \%, p=0.02)$ than in the control group (Figures $3 \mathrm{~B}, \mathrm{~F}$ ). Plasma HDL-C and TG levels did not differ between control and vaccinated groups (Figures 3 C, D).

\section{Long-lasting impact of liposomal vaccines in hypercholesterolemic mice}

A long-term follow-up was performed to evaluate the durability of antibody response against PCSK9 as well as the alterations in plasma cholesterol levels in hypercholesterolemic vaccinated mice. Analysis of antibody titers during 16 weeks after immunization with L-IFPTA ${ }^{+}$, L-IFPT, and IFPTA $^{+}$vaccine formulations revealed that a longlasting humoral immune response was persistently induced against PCSK9 peptide (Figure 2 A), which was correlated with a significant reduction of TC in L-IFPTA ${ }^{+}$, L-IFPT, and IFPTA ${ }^{+}$vaccine groups by up to $-82.5 \pm 7.3 \%(95 \% \mathrm{Cl}: 75-89 \%$, $p=0.002),-70.54 \pm 6.2 \%(95 \% \mathrm{Cl}: 65-76 \%, p=$ $0.013), 72.02 \pm 8.7 \%$ (95\% Cl: 64-79\%, $p=0.004)$, respectively (Figure $2 \mathrm{~B}$ ), and LDL-C up to $-88.14 \pm$ $5.6 \%$ (95\% Cl: $84-93 \%, p=0.002$ ), $-55.92 \pm 8.3 \%$, (95\% Cl: $48-63 \%, p=0.003)$ and $54.81 \pm 9.3 \%$ (95\% Cl: 46-63\%, $p=0.003$ ), respectively (Figure $2 \mathrm{C})$, compared to the pre-vaccination time point (WO) adjusted to the control group.

\section{Inverse association of PCSK9 antibody titers and LDL-C levels in vaccinated mice}

The statistical analysis revealed a significant negative association between plasma concentrations of LDL-C and titers of PCSK9 antibodies in the hypercholesterolemic vaccinated mice, in which LDL-C and PCSK9 antibodies were respectively at the lowest and highest levels. Therefore, the anti-PCSK9 vaccine formulations, to a higher extent than the L-IFPTA ${ }^{+}$vaccine, provoked high titers of PCSK9 antibodies that are directly associated with the decrease in plasma concentrations of LDL-C.

\section{Liposomal vaccines increase the hepatic LDLR}

Western blot and immunohistochemical (IHC) analyses were performed to evaluate the impact of liposomal anti-PCSK9 vaccine on the expression and cellular distribution of the hepatic LDLR protein in hypercholesterolemic C $57 \mathrm{BL} / 6$ mice (Figure 4). The hepatic LDLR protein was detected by Western blotting followed by quantification using ImageJ (Figure $4 \mathrm{~A}$ ). When normalized to $\beta$-actin, the protein expres sion of hepatic LDLR in hypercholesterolemic mice vaccinated with L-PTA ${ }^{+}$, L-IFPT, and IFPTA was found to be $2.93 \pm 0.32(95 \% \mathrm{Cl}: 2.1-3.7$, $p=0.0001), \quad 1.55 \pm 0.4(95 \% \quad \mathrm{Cl}: 0.4-2.4$ $p=0.03)$, and $1.39 \pm 0.4(95 \% \mathrm{Cl}: 0.2-2.2$, $p=0.06)$ fold greater than in the negative control mice (Figure $4 \mathrm{~B}$ ). As revealed by IHC analyses of the same liver samples, approximately similar staining of the cell membrane and cytoplasm was detected in the negative control group (Figure $4 \mathrm{C}$ ), while samples from vaccinated mice showed considerably increased staining at the cell membrane (Figure $4 \mathrm{D}$ ). Hence, nanoliposomal vaccines can efficiently increase the cell surface levels of hepatic LDLR in vaccinated hypercholesterolemic mice.

\section{Liposomal vaccine prevents aortic atherosclerotic lesions}

To evaluate the protective impact of the liposomal anti-PCSK9 vaccine on atherosclerosis lesion progression, lesion size, intima-to-media thickness (IMT), and lesion grade were assessed in the aortic arc upon a 16-week atherogenic diet (Figure 5). Mice immunized with the L-IFPTA vaccine exhibited a significantly decreased lesion size $(24.25 \%, p=0.002)$ and IMT $(30.2 \%$, $p=0.007)$, compared with the negative controls (Figures $5 \mathrm{~A}, \mathrm{~B}$ ). Moderate lesions (grade II) were observed in $57.2 \%$ and $28.5 \%$ of L-IFPTA+ and control mice, respectively; however, more severe lesions (grade III-IV) were only detected in control mice $(71.5 \%)$, while $42.85 \%$ of L-IFPTA ${ }^{+}$mice demonstrated grade I lesions (Figure $5 \mathrm{C}$ ). Histological images of H\&E-stained aortic arch lesions of vaccinated and negative control mice are presented in Figure 5 D. A significant and strong positive association was found between the size of aortic atherosclerotic lesions and plasma levels of LDL-C $(r=0.83, p=0.001)$ and PCSK9 protein $(r=0.71, p=0.0024)$.

\section{Liposomal vaccine exerts anti-inflammatory impact in hypercholesterolemic mice}

Flow cytometry analysis of splenocytes indicated that the L-IFPTA ${ }^{+}$vaccine was immunologically safe and increased anti-inflammatory response in hypercholesterolemic mice.

The L-IFPTA ${ }^{+}$vaccine could increase the antiinflammatory Th2 cell population (Figures $6 \mathrm{~A} 1$, 
A

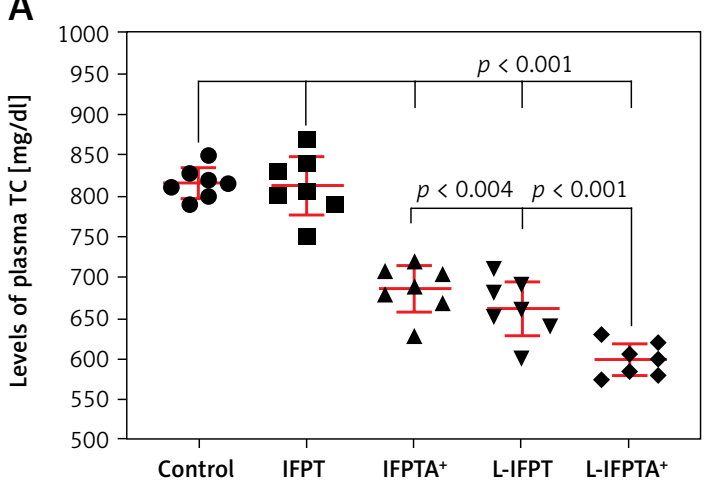

$\mathrm{C}$

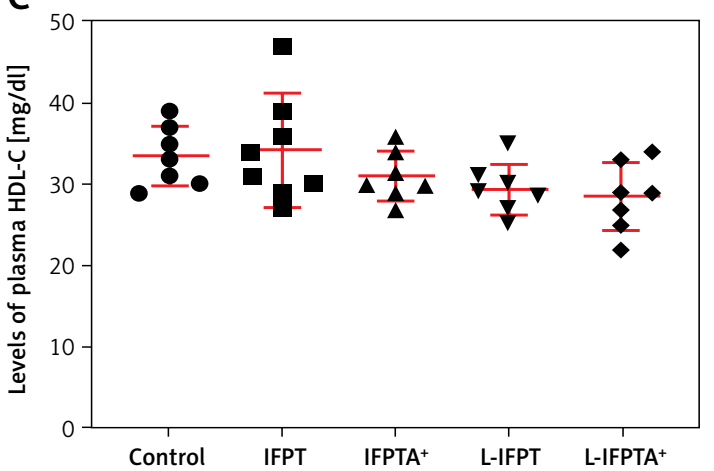

$E$

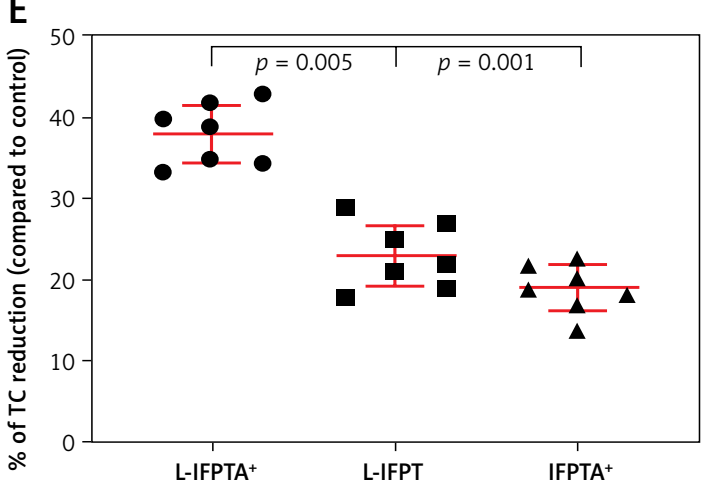

B

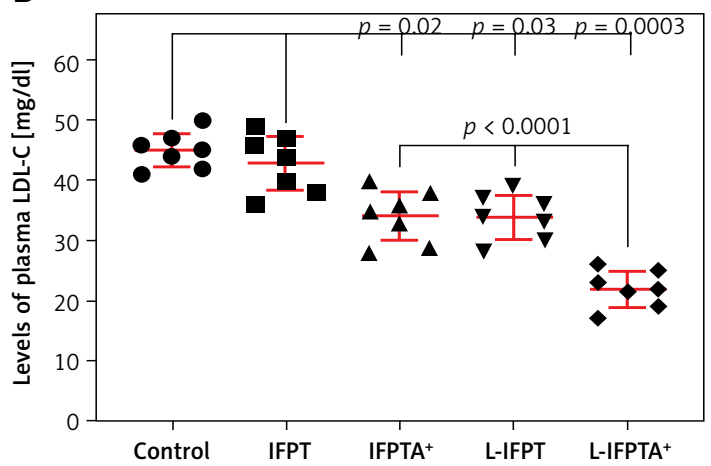

D

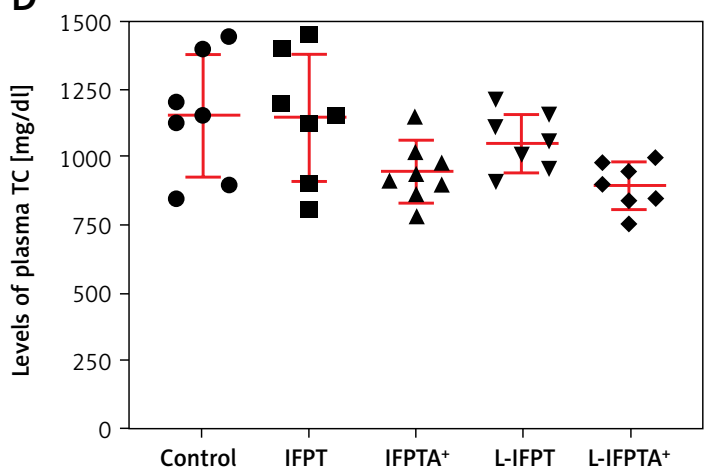

$\mathrm{E}$

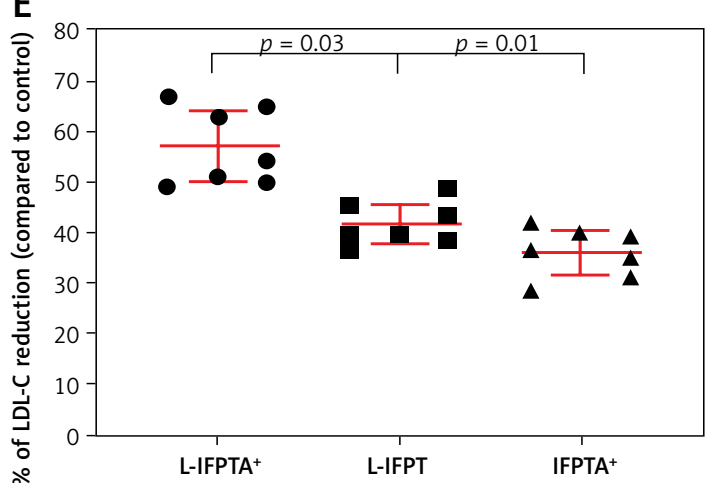

Figure 3. Lipid-lowering effect of anti-PCSK9 vaccine formulations. Levels of plasma TC (A), LDL-C (B), HDL-C (C), and TG (D) in hypercholesterolemic C57BL/6 mice, 2 weeks after the fourth vaccination (week 8) with various anti-PCSK9 vaccine formulations. Compared with the control group, TC $(E)$ and LDL-C (F) levels were significantly decreased in the L-IFPTA ${ }^{+}$, L-IFPT, and IFPTA ${ }^{+}$vaccine group 2 weeks after the last vaccination. There was no significant difference of HDL-C and TG between the control and the vaccinated groups. Data are shown as means with $95 \%$ confidence interval ( $n=7$ per group). One-way ANOVA followed by Tukey's post-hoc test was used to analyze the significance of the difference among groups. Statistical differences at $p$-values less than 0.05 were considered to be significant

HDL-C - high-density lipoprotein cholesterol, LDL-C - low-density lipoprotein cholesterol, TC - total cholesterol, TG - triglyceride.

A3) and corresponding IL-4 cytokine in vaccinated mice (Figures 6 B1, B3), whereas the baseline frequency of the pro-inflammatory Th1 cell population (Figures 6 A2, A4) and INF- $\gamma$ cytokine level (Figures 6 B2, B4) were not significantly changed. Compared with the control naïve mice, the population of Th2 cells (Figure 6 A3) and production of IL-4 cytokine (Figure 6 B3) were 1.76- and 4.9-fold higher in the L-IFPTA+ vaccine group.

\section{Discussion}

The current research demonstrated that active immunotherapy against PCSK9 using a nanoliposomal peptide-based vaccine termed L-IFPTA ${ }^{+}$could protect against hypercholesterolemia and atherosclerosis in mice immunized with a prophylactic route. Nanoliposomal anti-PCSK9 vaccine promoted functional and specific antibodies that targeted 
A

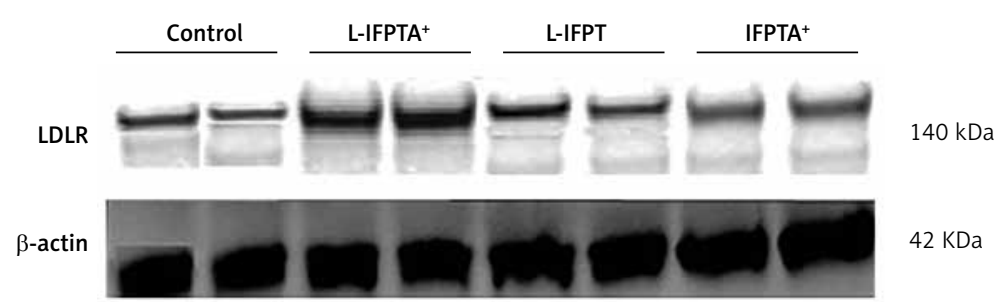

B

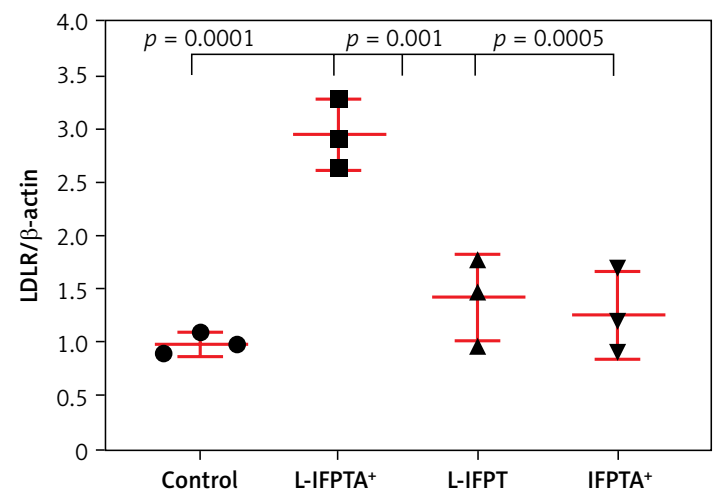

C

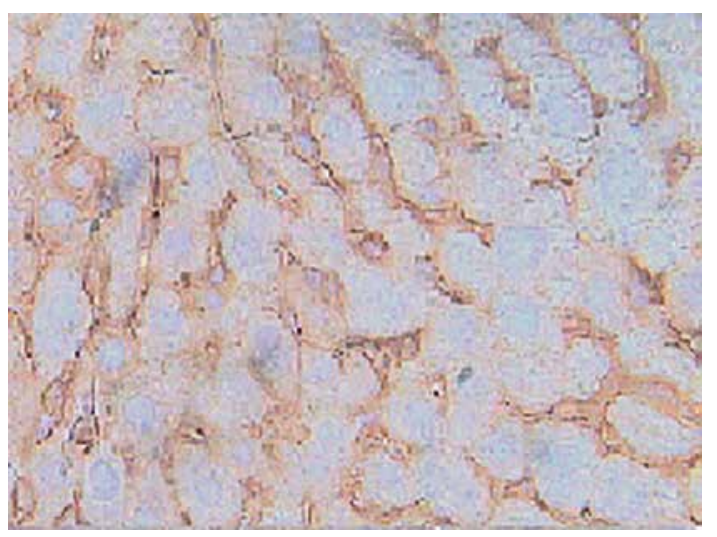

D

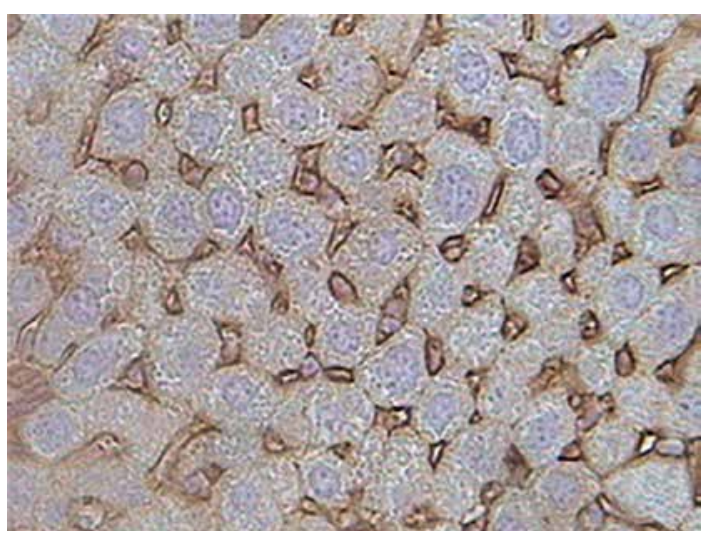

Figure 4. Expression (A, B) and cellular distribution (C, D) of LDLR in livers of vaccinated mice. Liver extracts from mice vaccinated with L-IFPTA ${ }^{+}$, L-IFPT, and IFPTA ${ }^{+}$, or from unvaccinated (control) mice were separated by SDS-PAGE and blotted onto a nitro-cellulose filter. Following incubation with HRP-conjugated anti-LDLR or anti- $\beta$ actin antibody, the bands corresponding to LDLR or $\beta$-actin proteins were measured by ImageJ software. The LDLR bands were normalized on the corresponding $\beta$-actin band (A). Compared to the control group, the protein expression of LDLR in the liver was significantly increased in the L-IFPTA ${ }^{+}$vaccine group. Data are expressed as means with $95 \%$ confidence interval ( $n=3$ per group). One-way ANOVA followed by Tukey's post-hoc test was used to determine the significance of the difference among groups. Statistical differences at $p$-values less than 0.05 were considered to be significant (B). Cellular distribution of LDLR in immunized mice (C, D). Immuno-histochemical analysis was carried out on liver samples isolated from the control (C) or vaccinated (D) mice using rabbit anti-LDLR antibodies detected by anti-rabbit IgG HRP-conjugated antibodies

circulating PCSK9 and suppressed its interaction with LDLR, resulting in a significant elevation of liver LDLR and subsequent reduction of TC and LDL-C. Additionally, significant reductions of inflammatory cells (Th1) and IFN- $\gamma$ cytokine as well as significant elevations of anti-inflammatory cells (Th2) and IL-4 cytokine were observed, which were parallel to the reduction of aortic atherosclerosis lesion size in hypercholesterolemic C57BL/6 mice.

PCSK9 has a pivotal role in LDL-C metabolism through promoting degradation of liver LDLR, which is the main route for clearance of circulating LDL-C. PCSK9 inhibition is considered to be the strongest therapeutic approach for reducing plasma LDL-C and atherosclerotic events. Evolocumab and alirocumab are two FDA-approved PCSK9 mAbs that were recommended by the 2016 ESC/EAS Guideline as second-line treatment for the management of dyslipidemias in hypercholesterolemic patients with an inadequate response to statin therapy [32]. Nevertheless, mAbs possess intrinsic disadvantages including 
A

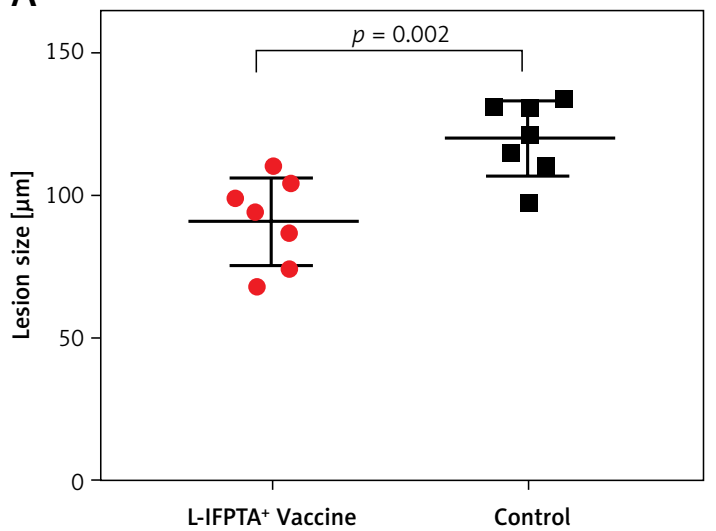

C

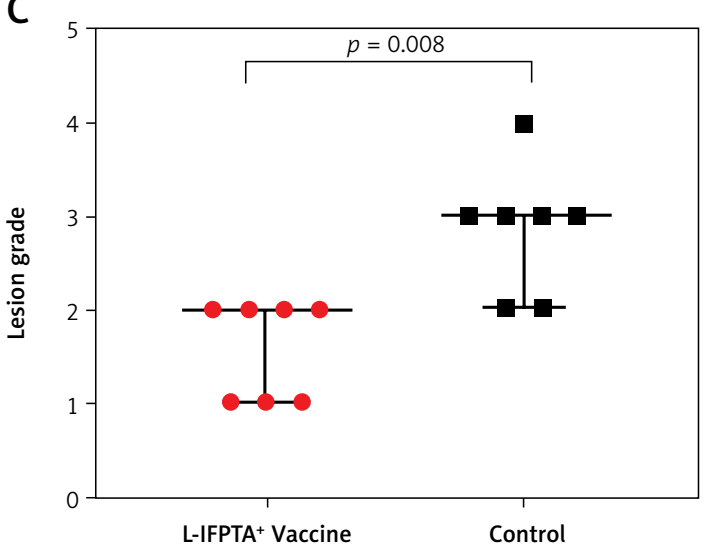

D

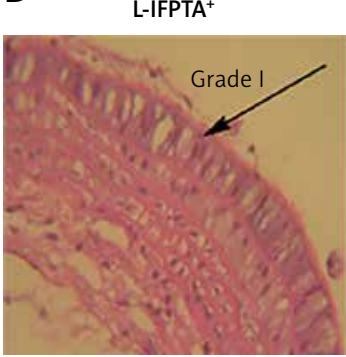

B

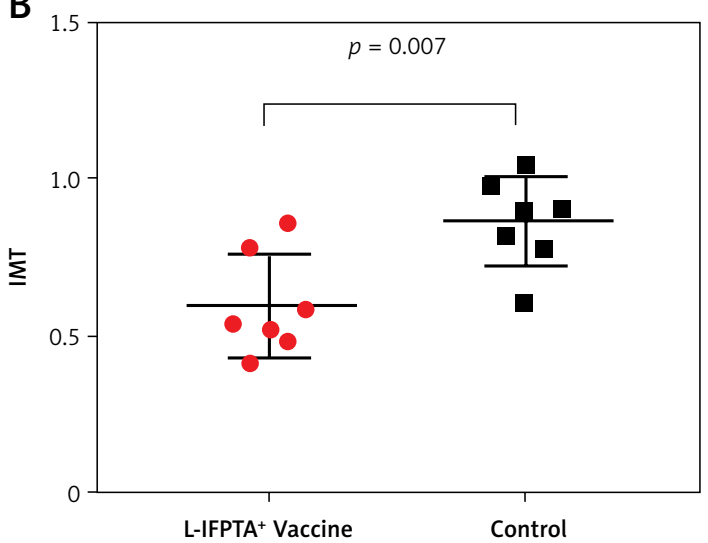

Figure 5. Inhibitory effect of L-IFPTA ${ }^{+}$vaccine on development of atherosclerotic lesion in aortic arc area of $\mathrm{C} 57 \mathrm{BL} / \mathrm{I}$ mice model of diet induced-atherosclerosis. 16 weeks after prime vaccination of mice on severe atherogenic diet lesion development was determined using hematoxylin and eosin (H\&E) staining. A - Lesion size in vaccinated and control mice was $90.86 \pm 5.849 \mu \mathrm{m}$ and $120 \pm 4.995 \mu \mathrm{m}$, respectively. Lesion size was found to be $24.25 \%(-29.14 \pm 7.692 \mu \mathrm{m}, p=0.002)$ lower in vaccinated mice than control mice. Data are expressed as group means \pm SD ( $n=7$ mice per group). Significance compared to control values was analyzed by unpaired 2-tailed Student's $t$-test. B - Intima-to-media thickness (IMT) in vaccinated and control mice was $0.5978 \pm 0.06271$ and 0.8634 \pm 0.05393 , respectively. IMT in vaccine group was significantly decreased by $30.2 \%(0.2656 \pm 0.08271 \mu \mathrm{m}, p=$ 0.007 ) compared with control group. Data are expressed as group means \pm SD ( $n=7$ mice per group). Significance compared to control values was analyzed by unpaired 2-tailed Student's t-test. C - Lesion grade in vaccinated and control mice was 2 [1-2] and 3 [2-3], respectively. Data are expressed as group medians [ $25^{\text {th }}$ percentile $-75^{\text {th }}$ percentile] ( $n=7$ mice per group). Significance compared to control values was analyzed by Mann-Whitney $U$ test. Statistical differences at $p$-values less than 0.05 were considered to be significant. D - Representative images of H\&E-stained grade I, II, III, and IV lesions of aortic arc

high-dose and frequent administration [33], possible induction of host anti-mAb antibodies [34], and high cost [35], which restrict their clinical use for chronic diseases demanding long-term treatments. To overcome such drawbacks, active immunotherapy with long-lasting effects can be a cost-effective and safe alternative to mAb- based therapy of hypercholesterolemia and atherosclerosis [34, 36].

In a previous study, we demonstrated that our liposomal peptide-based active immunization approach could strongly elicit a durable and safe PCSK9-specific antibody response in vaccinated $\mathrm{BALB} / \mathrm{C}$ mice [22]. Accordingly, it was shown that 
A1

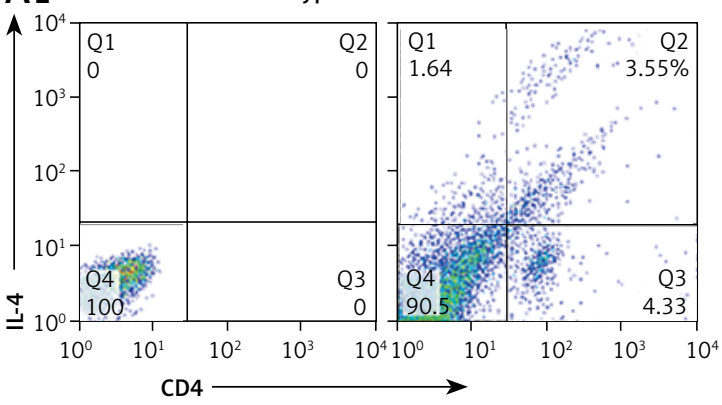

A3

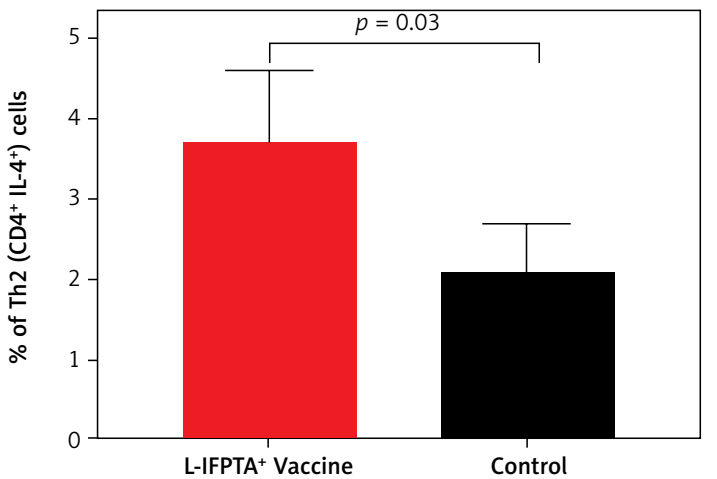

A2 Isotype

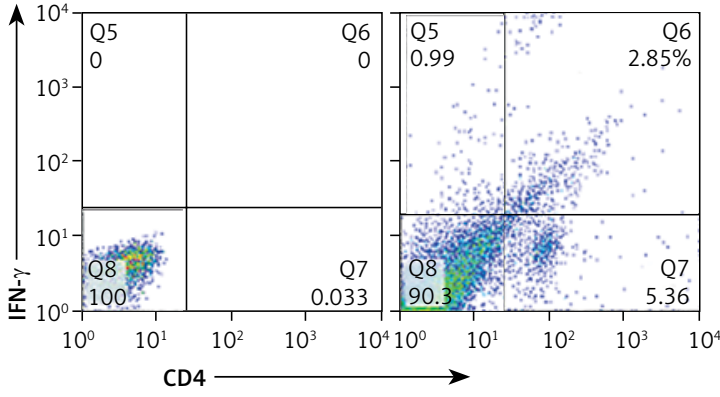

A4

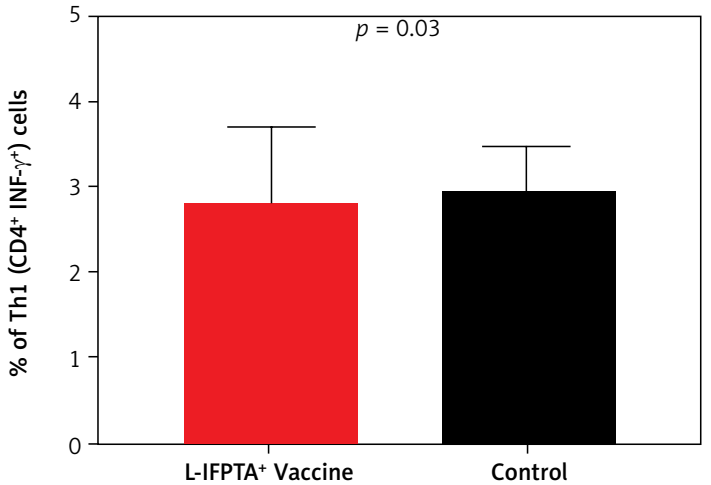

Figure 6. A - L-IFPTA ${ }^{+}$vaccine increase anti-inflammatory Th2 cells in hypercholesterolemic C57BL/6 mice. Representative scattergrams exhibit gating strategy and quadrant analysis for flow cytometry of Th2 (CD4 $4^{+}$IFN- $\gamma^{+}$ T cell) (A1) and Th1 (CD4+ IL-4+ T cell) (A2) population on the basis of isotype control. Splenic Th2 cell population in L-IFPTA $^{+}$vaccine group were higher than in naïve control mice (A3), while there was no significant difference in Th1 cell population of vaccine and control group (A4)

the liposomal anti-PCSK9 vaccine could strongly decrease LDL-C and inhibit progression of atherosclerotic lesion in C57BL/6 mice on a harsh atherogenic regimen [23]. Vaccine-based PCSK9 suppression has also been evaluated by others using different approaches. AFFiRiS developed an AFFITOPE-based PCSK9 active immunization approach [14] that decreased TC by $53 \%$, which was correlated with decreased size and severity of atherosclerosis plaque in vaccinated compared with control mice [15]. Likewise, Fattori et al. studied a combination of human recombinant PCSK9 and DNA oligonucleotide as an adjuvant, and observed an approximately $40 \%$ decrease of LDL-C in vaccinated mice [16]. Pan et al. developed bacteriophage virus-like particles, on top of which PCSK9 peptides were displayed at a high valency. This human PCSK9 vaccine reduced TC levels by $\sim 20 \%$ compared with the control [17].

We are the first to present the liposomal antiPCSK9 vaccine L-IFPTA ${ }^{+}$that exhibits prophylactic effects against hypercholesterolemia and atheroma formation in a C57BL/6 mouse model of severe atherosclerosis. The L-IFPTA $^{+}$vaccine elicits high-titer specific antibodies by which plasma levels of PCSK9 in hypercholesterolemic mice were decreased by up to $40 \%$, compared with the control mice. Target engagement analysis in vivo revealed that L-IFPTA ${ }^{+}$-induced antibodies bound specifically to plasma PCSK9 in vaccinated mice. In vitro analysis of PCSK9-LDLR interaction demonstrated that the L-IFPTA ${ }^{+}$vaccine generated functional PCSK9 antibodies that inhibited interaction between PCSK9 and LDLR. It is well documented that inhibition of the PCSK9-LDLR interaction causes up-regulation of LDLR on the surface of hepatocytes, thereby enhancing the uptake of plasma cholesterol from the bloodstream [16, 37]. In line with this evidence, our approach inhibited the PCSK9-LDLR interaction, which consequently resulted in LDLR up-regulation and subsequent reduction of plasma cholesterol, particularly LDL-C. The L-IFPTA ${ }^{+}$vaccine inhibited the increase of plasma TC and LDL-C as well as progression of atherosclerotic lesion in C57BL/6 mice on a severe atherogenic diet plus poloxamer 407 injection. Two weeks after the fourth vaccination (week 8), the mean PCSK9 antibody titers reached the highest levels in hypercholesterolemic mice and resulted in reductions of TC and LDL-C by up to $-38.13 \%$ and $-55 \%$, respectively. The results are supported by statistical analysis that showed a significant inverse correlation between plasma levels of LDL-C and PCSK9 antibody titers. Long-term monitoring (16 weeks) showed that during 10 weeks after the last vaccination (weeks 6-16) the antibody 
B1
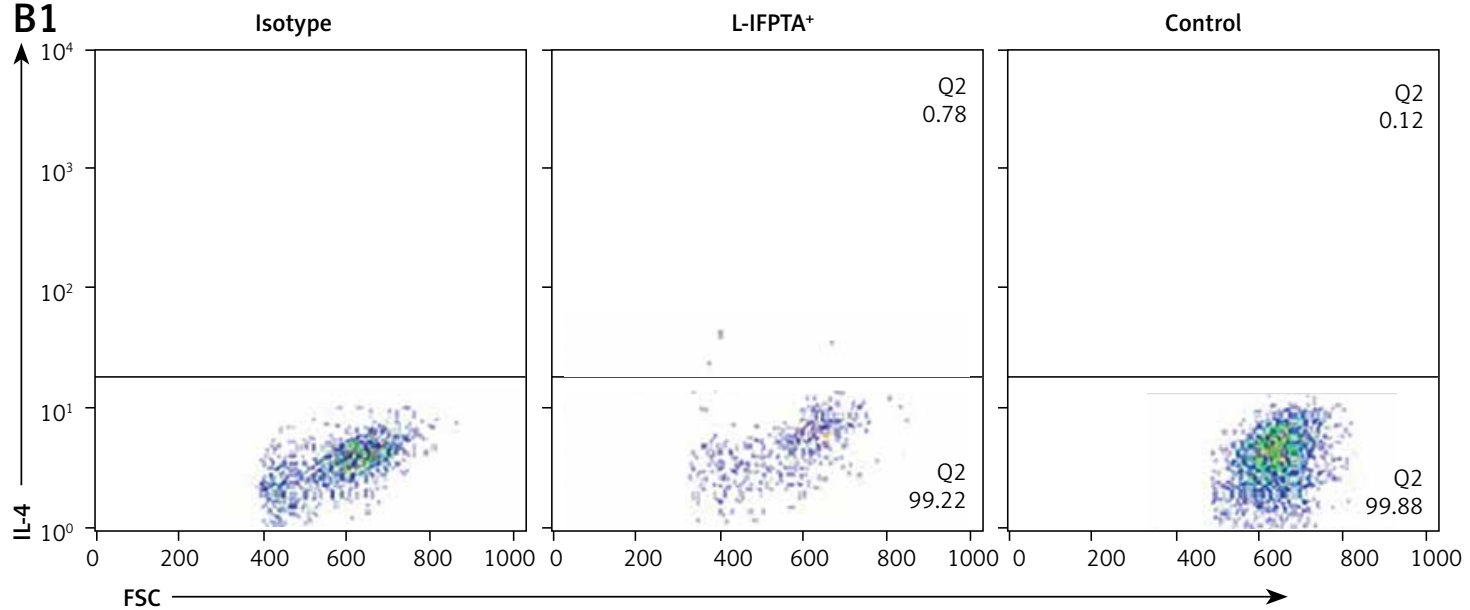

B2 Isotype L-IFPTA $^{+}$
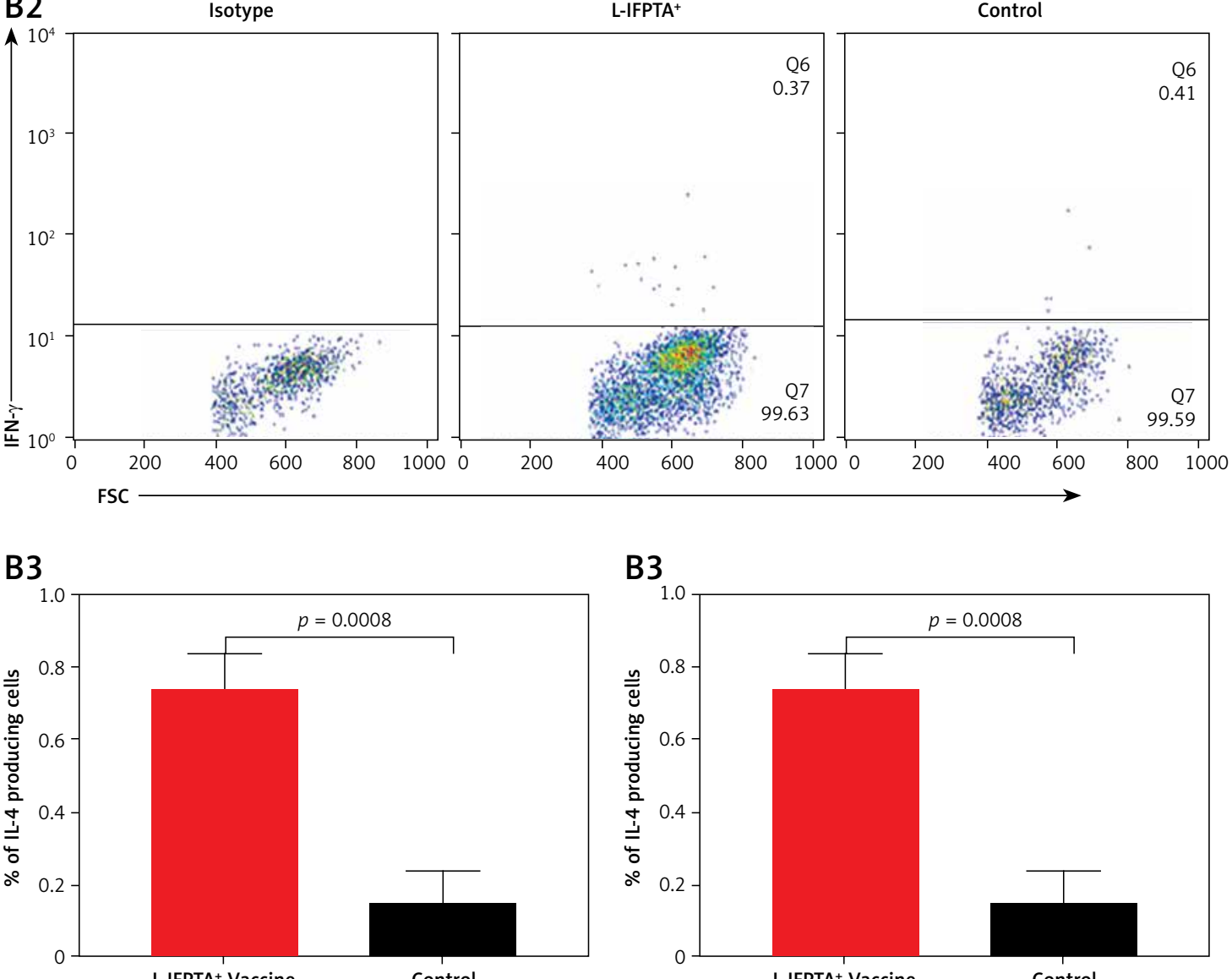

B3

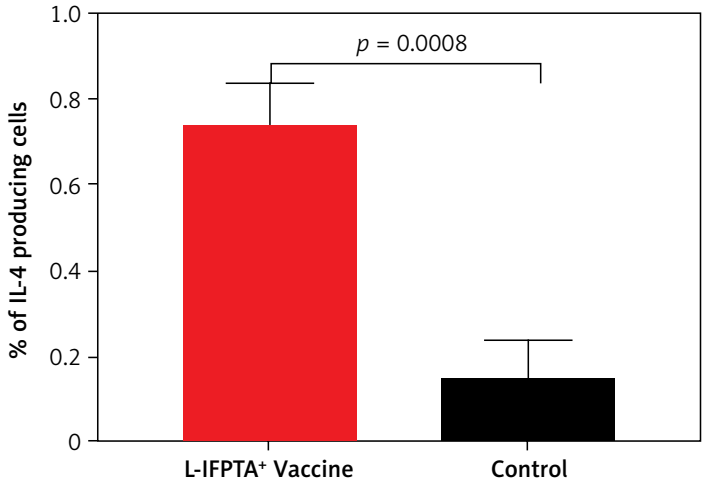

B3

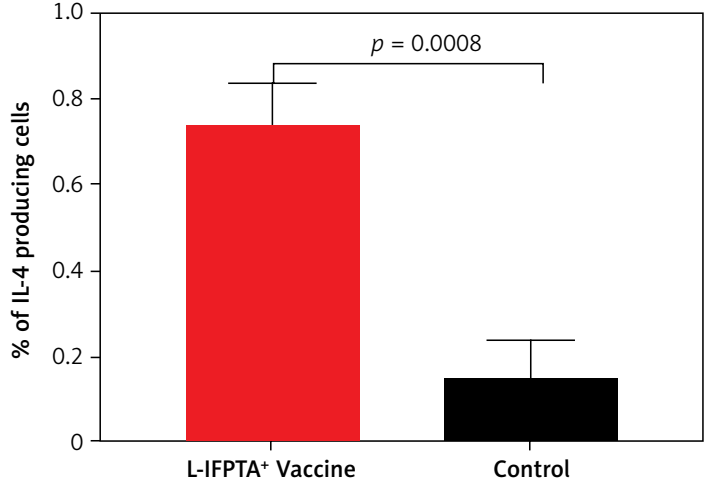

Figure 6. Cont. B - L-IFPTA ${ }^{+}$vaccine increased anti-inflammatory IL-4 cytokine in hypercholesterolemic C57BL/6 mice. Representative scattergrams exhibit gating strategy and quadrant analysis for flow cytometry of IL-4 (B1) and IFN- $\gamma^{+}$(B2) producing splenocytes on the basis of isotype control. Expression of IL-4 in L-IFPTA ${ }^{+}$vaccine group was significantly higher than in naïve control mice (B3), while there was no significant difference of IFN- $\gamma^{+}$between vaccine and control groups (B4). Data are expressed as mean \pm SD of 7 mice in each group. Significance compared to control values was analyzed by unpaired 2-tailed Student's $t$-test. Statistical differences at $p$-values less than 0.05 were considered to be significant

titer in vaccinated mice remained significantly higher than that in the control group, which was accompanied by significant reductions in TC and LDL-C at these time points. Adjusting data to the control and comparing them to the onset time point revealed that at the end of the study (during 16 weeks) TC was decreased by up to $-82.5 \%$ and LDL-C up to $-88.14 \%$ in vaccinated mice on a severe atherogenic diet, which was associated with significantly lower lesion size $(-24.25 \%, p=0.002)$ 
and severity $(-45 \%, p=0.008)$ in the aortic arch compared with the control.

An inhibitory effect of the PCSK9 vaccine on progression of atherosclerotic lesions was also observed in $\mathrm{APOE}^{\star} 3$ Leiden.CETP mice [15]; however, our study demonstrated the atheroprotective effect of L-IFPTA ${ }^{+}$in mice receiving a concomitant atherogenic diet plus poloxamer 407 injection to induce severe atherosclerosis. This model has repeatedly been shown to be reliable for studying atherosclerosis and its pathological features in mice [38]. Nevertheless, an important limitation for developing PCSK9 inhibitors is the lack of a reliable mouse model that can recapitulate high levels of LDL-C in humans. Indeed, in the mouse, unlike the human, a large amount of cholesterol is eliminated by HDL metabolism, and thus baseline levels of LDL-C are not sufficiently increased as those witnessed in human [38]. Therefore, this can reveal a possible reason for the moderately lower effectiveness of PCSK9 inhibitors when trialed in humans $[14,15]$. For example, despite the efficient LDL-C lowering of an anti-PCSK9 vaccine in preclinical studies, its effect was less impressive, with a $13 \% \mathrm{LDL}-\mathrm{C}$ level reduction, when tested in a phase I clinical trial (Bauer et al., Communication at ESC Congress, 2018 Munich). Notably, although in the present study we designed a preventive approach in which baseline level of LDL-C was low in hypercholesterolemic mice when vaccination was primed, in our previous study using the therapeutic approach, an elevated level of baseline LDL-C ( $200 \mathrm{mg} / \mathrm{dl})$ was evidenced [23], suggesting that the hypercholesterolemic mouse model developed in this study may be a model near to human hypercholesterolemia and hence can provide a reliable model that extrapolates preclinical data of anti-PCSK9 inhibitors to humans. Thus, the preventive effect of the L-IFPTA ${ }^{+}$vaccine observed in the current study can introduce a promising strategy for effective prevention of increasing levels of LDL-C and atherosclerotic plaque progression in humans.

Inflammation is known to have an important role in atherosclerosis progression and complications. T lymphocytes, particularly subsets of CD4+ helper T (Th) cells, have been found to play an important role in atherosclerosis, both in humans [39] and in animals [40]. These CD4-bearing Th cells are classified mainly into Th1 $\left(\mathrm{CD}^{+} \mathrm{IFN}-\gamma^{+}\right)$ and Th2 $\left(\mathrm{CD}^{+} \mathrm{IL}^{+} 4^{+}\right)$subtypes showing pro-atherogenic and anti-atherogenic roles, respectively [41-43]. The pro-inflammatory IFN- $\gamma$ cytokine can induce and worsen atherosclerosis progression via influencing lipid accumulation and foam cell formation in the vascular wall, and cellular composition of the plaque, such as inducing proliferation of SMCS, macrophage activation, autocrine induction of Th1 cells, and suppressing proliferation of Th2 cells [44]. In contrast, IL-4 secreted by Th2 cells counteracts the IFN- $\gamma$ production [45]. Further, high levels of Th2 cells in the circulating blood were independently associated with a decrease in carotid plaque thickness, and a reduced risk of acute myocardial infarction in females [45]. In the present study, it was found that the population of anti-inflammatory Th2 cells as well as production of IL-4 cytokine were significantly increased by 1.76 - and 4.9-fold, respectively, in splenic cells isolated from the L-IFPTA ${ }^{+}$-vaccinated mice compared with controls. This finding implies that the L-IFPTA ${ }^{+}$vaccine induced a safe immune response in hypercholesterolemic mice.

In conclusion, the L-IFPTA ${ }^{+}$vaccine induced safe, durable and functional PCSK9-specific antibodies in hypercholesterolemic C57BL/6 mice. This resulted in a long-term therapeutic, as reported previously [23], and protective effects against hypercholesterolemia and atherosclerosis. The present findings suggest the potential of the L-IFPTA $^{+}$vaccine as a potentially effective alternative to $\mathrm{mAb}$-based therapies for controlling elevated cholesterol levels and preventing cardiovascular disease.

\section{Conflict of interest}

The authors declare no conflict of interest.

\section{References}

1. Kannel WB, Dawber TR, Friedman GD, Glennon WE, MCnamara PM. Risk factors in coronary heart disease: an evaluation of several serum lipids as predictors of coronary heart disease: the Framingham study. Ann Intern Med 1964; 61: 888-99.

2. Robinson JG, Rosenson RS, Farnier M, et al. Safety of very low low-density lipoprotein cholesterol levels with alirocumab: pooled data from randomized trials. J Am Coll Cardiol 2017; 69: 471-82.

3. Giugliano RP, Wiviott SD, Blazing MA, et al. Long-term safety and efficacy of achieving very low levels of low-density lipoprotein cholesterol: a prespecified analysis of the IMPROVE-IT trial. JAMA Cardiol 2017; 2: 547-55.

4. Group SSSS. Randomised trial of cholesterol lowering in 4444 patients with coronary heart disease: the Scandinavian Simvastatin Survival Study (4S). Lancet 1994; 344: 1383-9.

5. Blom DJ, Djedjos CS, Monsalvo ML, et al. Effects of evolocumab on vitamin $\mathrm{E}$ and steroid hormone levels: results from the 52-week, phase 3, double-blind, randomized, placebo-controlled DESCARTES study. Circ Res 2015; 117: 731-41.

6. Sabatine MS, Giugliano RP, Wiviott SD, et al. Efficacy and safety of evolocumab in reducing lipids and cardiovascular events. N Engl J Med 2015; 372: 1500-9.

7. Robinson JG, Farnier M, Krempf M, et al. Efficacy and safety of alirocumab in reducing lipids and cardiovascular events. N Engl J Med 2015; 372: 1489-99.

8. Sabatine M, Giugliano R, Keech A, et al. FOURIER Steering Committee and Investigators. Evolocumab and clin- 
ical outcomes in patients with cardiovascular disease. N Engl J Med 2017; 376: 1713-22.

9. Nicholls SJ, Puri R, Anderson T, et al. Effect of evolocum$a b$ on progression of coronary disease in statin-treated patients: the glagov randomized clinical trial. JAMA 2016; 316: 2373-84.

10. Catapano A, Papadopoulos N. The safety of therapeutic monoclonal antibodies: implications for cardiovascular disease and targeting the PCSK9 pathway. Atherosclerosis 2013; 228: 18-28.

11. Do RQ, Vogel RA, Schwartz GG. PCSK9 Inhibitors: potential in cardiovascular therapeutics. Curr Cardiol Rep 2013; 15: 345.

12. Hall SS. A gene of rare effect. Nature 2013; 496: 152-5.

13. Sahebkar A, Watts GF. New therapies targeting apoB metabolism for high-risk patients with inherited dyslipidaemias: what can the clinician expect? Cardiovasc Drugs Ther 2013; 27: 559-67.

14. Galabova G, Brunner S, Winsauer G, et al. Peptide-based anti-PCSK9 vaccines-an approach for long-term LDLC management. PLoS One 2014; 9: e114469.

15. Landlinger C, Pouwer MG, Juno C, et al. The AT04A vaccine against proprotein convertase subtilisin/kexin type 9 reduces total cholesterol, vascular inflammation, and atherosclerosis in $\mathrm{APOE}^{*}$ 3Leiden. CETP mice. Eur Heart 2017; 38: 2499-507.

16. Fattori E, Cappelletti M, Surdo PL, et al. Immunization against proprotein convertase subtilisin-like/kexin type 9 (PCSK9) lowers plasma LDL-cholesterol levels in mice. J Lipid Res 2012; 53: 1654-61.

17. Pan Y, Zhou Y, Wu H, et al. A therapeutic peptide vaccine against PCSK9. Sci Rep 2017; 7: 12534.

18. Slingluff CL, Yamshchikov G, Neese P, et al. Phase I trial of a melanoma vaccine with gp100280-288 peptide and tetanus helper peptide in adjuvant: immunologic and clinical outcomes. Clin Cancer Res 2001; 7: 3012-24.

19. Bachmann MF, Dyer MR. Therapeutic vaccination for chronic diseases: a new class of drugs in sight. Nature Rev Drug Discov 2004; 3: 81-8.

20. Nakagami H, Koriyama H, Morishita R. Therapeutic vaccines for hypertension and dyslipidemia. Int Heart 2014; 55: 96-100.

21. Zamani P, Momtazi-Borojeni AA, Nik ME, Oskuee RK, Sahebkar A. Nanoliposomes as the adjuvant delivery systems in cancer immunotherapy. J Cell Physiol 2018; 233: 5189-99.

22. Momtazi-Borojeni AA, Jaafari MR, Badiee A, Sahebkar A. Long-term generation of antiPCSK9 antibody using a nanoliposome-based vaccine delivery system. Atherosclerosis 2019; 283: 69-78.

23. Momtazi-Borojeni AA, Jaafari MR, Badiee A, Banach M, Sahebkar A. Therapeutic effect of nanoliposomal PCSK9 vaccine in a mouse model of atherosclerosis. BMC Med 2019; 17: 223.

24. O'Keeffe LM, Simpkin AJ, Tilling K, et al. Sex-specific trajectories of measures of cardiovascular health during childhood and adolescence: a prospective cohort study. Atherosclerosis 2018; 278: 190-6.

25. Herrington W, Lacey B, Sherliker P, Armitage J, Lewington $\mathrm{S}$. Epidemiology of atherosclerosis and the potential to reduce the global burden of atherothrombotic disease. Circ Res 2016; 118: 535-46.

26. Libby P, Buring JE, Badimon L, et al. Atherosclerosis. Nat Rev Dis Primers 2019; 5: 56.

27. Guillén J, Prins JB, Howard B, Degryse AD, Gyger M. The European framework on research animal welfare regulations and guidelines. Laboratory animals: regulations and recommendations for the care and use of animals in research Academic Press UK 2018; 117-202.

28. Close B, Banister K, Baumans V, et al. Recommendations for euthanasia of experimental animals: Part 2. DGXT of the European Commission. Lab Anim 1997; 31: 1-32.

29. Close B, Banister K, Baumans V, et al. Recommendations for euthanasia of experimental animals: Part 1. Lab Anim 1996; 30: 293-316.

30. Johnston TP, Korolenko TA, Sahebkar A. P-407-induced mouse model of dose-controlled hyperlipidemia and atherosclerosis: 25 years later. J Cardiovasc Pharmacol 2017; 70: 339-52.

31. Chekanov VS, Mortada ME, Tchekanov GV, et al. Pathologic and histologic results of electrical impulses in a rabbit model of atherosclerosis: 24 -hour versus 8-hour regimen. J Vasc Surg 2002; 35: 554-62.

32. Catapano AL, Graham I, De Backer G, et al. 2016 ESC/ EAS guidelines for the management of dyslipidaemias. Eur Heart J 2016; 37: 2999-3058.

33. Maningat P, Gordon BR, Breslow JL. How do we improve patient compliance and adherence to long-term statin therapy? Curr Atheroscler Rep 2013; 15: 291.

34. Bartelds GM, Krieckaert CL, Nurmohamed MT, et al. Development of antidrug antibodies against adalimumab and association with disease activity and treatment failure during long-term follow-up. JAMA 2011; 305: 1460-8.

35. Shrank W, Lotvin A, Singh S, Brennan T. In the debate about cost and efficacy, PCSK9 inhibitors may be the biggest challenge yet. Health Affairs 2015.

36. Chackerian B, Remaley A. Vaccine strategies for lowering LDL by immunization against proprotein convertase subtilisin/kexin type 9. Curr Opin Lipidol 2016; 27: 345-50.

37. Zhang L, McCabe T, Condra JH, et al. An anti-PCSK9 antibody reduces LDL-cholesterol on top of a statin and suppresses hepatocyte SREBP-regulated genes. Int I Biol Sci 2012; 8: 310-27.

38. Camus M, Chapman M, Forgez P, Laplaud P. Distribution and characterization of the serum lipoproteins and apoproteins in the mouse, Mus musculus. J Lipid Res 1983; 24: $1210-28$.

39. Stary HC, Chandler AB, Glagov S, et al. A definition of initial, fatty streak, and intermediate lesions of atherosclerosis. A report from the Committee on Vascular Lesions of the Council on Arteriosclerosis, American Heart Association. Circulation 1994; 89: 2462-78.

40. Zhou X, Stemme S, Hansson GK. Evidence for a local immune response in atherosclerosis. CD4+ T cells infiltrate lesions of apolipoprotein-E-deficient mice. Am J Pathol 1996; 149: 359-66.

41. Huber SA, Sakkinen P, David C, Newell M, Tracy R. T helper-cell phenotype regulates atherosclerosis in mice under conditions of mild hypercholesterolemia. Circulation 2001; 103: 2610-6.

42. Laurat E, Poirier B, Tupin E, et al. In vivo downregulation of $T$ helper cell 1 immune responses reduces atherogenesis in apolipoprotein E-knockout mice. Circulation 2001; 104: 197-202.

43. Tedgui A, Mallat Z. Cytokines in atherosclerosis: pathogenic and regulatory pathways. Physiol Rev 2006; 86: 515-81.

44. Baidya S, Zeng Q. Helper T cells and atherosclerosis: the cytokine web. Postgrad Med J 2005; 81: 746-52.

45. Wurtz O, Bajénoff M, Guerder S. IL-4-mediated inhibition of IFN- $\gamma$ production by $\mathrm{CD} 4+\mathrm{T}$ cells proceeds by several developmentally regulated mechanisms. Int Immunol 2004; 16: 501-8. 